Document downloaded from:

http://hdl.handle.net/10251/31823

This paper must be cited as:

California, A.; Cardoso, VF.; Costa, CM.; Sencadas, V.; Botelho, G.; Gómez Ribelles, JL.; Lanceros-Mendez, S. (2011). Tailoring porous structure of ferroelectric poly(vinylidene fluoride-trifluoroethylene) by controlling solvent/polymer ratio and solvent evaporation rate. EUROPEAN POLYMER JOURNAL. 47(12):2442-2450. doi:10.1016/j.eurpolymj.2011.10.005



The final publication is available at

http://dx.doi.org/10.1016/j.eurpolymj.2011.10.005

Copyright Elsevier

Additional Information 


\title{
Tailoring porous structure of ferroelectric poly(vinylidene fluoride- trifluoroethylene) by controlling solvent/polymer ratio and solvent evaporation rate
}

\author{
António California ${ }^{1}$; Vanessa F. Cardoso ${ }^{1,2}$, Carlos M. Costa ${ }^{1}$, Vitor Sencadas ${ }^{1}$; \\ Gabriela Botelho $^{3}$, José L. Gómez-Ribelles ${ }^{4,5,6}$ and Senentxu Lanceros-Mendez ${ }^{1, *}$ \\ ${ }^{1}$ Centro/Departamento de Física, Universidade do Minho, Campus de Gualtar, 4710-058 \\ Braga, Portugal \\ ${ }^{2}$ Department of Industrial Electronics, University of Minho, Campus de Azurém, 4800-058 \\ Guimarães, Portugal. \\ ${ }^{3}$ Centro /Departamento de Química, Universidade do Minho, 4710-057 Braga, Portugal \\ ${ }^{4}$ Center for Biomaterials and Tissue Engineering, Universidad Politécnica de Valencia, \\ Camino de Vera s/n, 46022 Valencia, Spain \\ ${ }^{5}$ Prince Felipe Research Center, Autopista del Saler 16, 46013 Valencia, Spain \\ ${ }^{6}$ Networking Research Center on Bioengineering, Biomaterials and Nanomedicine \\ (CIBER-BBN), Valencia, Spain
}

Email: lanceros@fisica.uminho.pt

\begin{abstract}
Ferroelectric macroporous poly(vinylidene fluoride-trifuoroethylene) membranes have been produced by isothermal crystallization from the solution at different temperatures starting from different diluted solutions of the co-polymer in dimethylformamide. In this way pore architecture, consisting in interconnected spherical pores can be obtained. The mechanism and kinetics of solvent evaporation was investigated and related to the evolution of the polymer microstructure. The mechanism underlying the pattern formation has been discussed on the light of the Flory-Huggins (FH) lattice teory, grain
\end{abstract}


boundary effects and the Cahn-Hilliard equation for mass conservation systems. It was also observed that the temperature or initial concentration of the crystallization process does not affect the phase, ferroelectric transition temperature or the melting temperature of the polymer.

Keywords: electroactive membranes, porous membranes, PVDF, solvent evaporation, phase diagram

\section{Introduction}

Poly(vinylidene fluoride), PVDF and VDF (vinylidene fluoride) copolymers like $\mathrm{P}(\mathrm{VDF}-\mathrm{TrFE})$ (Poly(vinylidene fluoride - co - trifluorethylene)) are polymer materials known for their interesting electroactive properties, which allow electro-optical, electromechanical and biomedical applications $[1,2]$. In particular, fabrication of porous membranes of these materials has attracted interest due to their potential applications as filters; as polymer electrolyte for applications in rechargeable batteries [3, 4]; and in biomedical applications $[5,6]$. Phase separation of the polymer solution for the preparation of porous membranes can be induced by several methods, including immersion precipitation, precipitation from the vapour phase, and thermally induced phase separation (TIPS) [7, 8]. Until now, only a few works have been reported on preparation of PVDF microporous membrane via TIPS [4, 7-10]. This processing technique has proven to be a valuable method for making commercial membranes as it shows advantages such as low tendency for defect formation, high degrees of porosity and effective control of the pore size. In the membrane preparation by TIPS, thermodynamic properties, such as the shape of the phase diagram can strongly affect the pore size and degree of porosity. Selecting the proper solvent is also one of the key 
factors for controlling the pore size as the compatibility of polymer and solvent affects the solution thermodynamic properties such as the binodal line and crystallization temperature. Furthermore, different quenching conditions also affect the polymer crystallization microstructure [10-12].

Through it has been previously proven that the isothermal evaporation from a homogeneous $\mathrm{P}(\mathrm{VDF}-\mathrm{TrFE})$ copolymer solution produce a well-organized porous structure in membranes from 1 to $250 \mu \mathrm{m}$ [13], the different experimetal conditions that can allow tayloring membrane characteristics have not been explored and the mechanism responsible for the pattern fomation has to be further explained. In the present work, that initial work has been systematically extended by preparing electroactive $\mathrm{P}(\mathrm{VDF}-\mathrm{TrFE})$ membranes with controlled porosity by solvent evaporation at different temperatures and from different polymer/solvent relative concentrations. Most important, the origin of the microstructure formation is investigated by relating it to the solvent evaporation kinetics. In this way, both a method for processing electroactive $\mathrm{P}(\mathrm{VDF}-\mathrm{TrFE})$ membranes and the understanding of the process have been achieved, which ensures reproducibility and further development.

\section{Experimental}

Poly(vinylidene fluoride-trifuoroethylene) (PVDF-TrEF) (72/28) (Solvay, Brussels, Belgium ) was dissolved in N,N-dimethyl formamide (DMF - Merck). After total dissolution of the polymer, the solution was placed in a $10 \mu \mathrm{L}$ pan with a micropipette (PIPP-003-500, Labbox) and then kept inside of a thermogravimetric oven (PerkinElmer Pyris-1 TGA) at isothermal temperature between 20 to $60{ }^{\circ} \mathrm{C}$. The initial polymer 
concentrations were 5, 10, 15 and $20 \%$ volume fractions. All experiments were carried out under nitrogen atmosphere with gas flow at $60 \mathrm{ml} / \mathrm{h}$ and balance $40 \mathrm{ml} / \mathrm{h}$.

The microstructure of the samples was measured by scanning electron microscopy (SEM) with a FEI Nova 200 apparatus at room temperature. The crystalline phase of the samples was confirmed by Fourier Transformed Infrared Spectroscopy (FTIR) (Spectrum 100) in range between $650-4000 \mathrm{~cm}^{-1}$. FTIR spectra were collected with 32 scans and a resolution of $4 \mathrm{~cm}^{-1}$. The thermal behavior of the samples was analyzed by differential scanning calorimetry (DSC) measurements with a Perkin Elmer Diamond DSC apparatus. The samples were cut into small pieces from the middle region of the membranes, placed into $40 \mu \mathrm{l}$ aluminum pans and heated between 30 and $200{ }^{\circ} \mathrm{C}$ at a heating rate of $10^{\circ} \mathrm{C} \cdot \mathrm{min}^{-1}$.

The porosity of the samples was estimated by pycnometry. The porosity ( $\varepsilon$ ) was measured by an improved weight-method [14]. The weight of the pycnometer, filled with ethanol, was measured $\left(\mathrm{W}_{1}\right)$; the sample, whose weight was $\mathrm{W}_{\mathrm{s}}$, was immersed in ethanol. After the pore of the scaffolds was saturated by ethanol, additional ethanol was added to complete the volume of the pycnometer. Then, the pycnometer was weighted $\left(\mathrm{W}_{2}\right)$; the sample filled with ethanol was taken out of the pycnometer. The residual weight of the ethanol and the pycnometer was labeled $\mathrm{W}_{3}$. The porosity of the scaffold was calculated according to:

$$
\varepsilon=\frac{W_{2}-W_{3}-W_{s}}{W_{1}-W_{3}}
$$

Mean porosity of each membrane was obtained as the average of the values determined in three samples and the results are presented in table 1. 
Table 1: Influence of the viscosity on the $\mathrm{P}(\mathrm{VDF}-\mathrm{TrFE})(72 / 28) / \mathrm{DMF}$ samples porosity obtained by solvent evaporation at $20^{\circ} \mathrm{C}$.

\begin{tabular}{|c|c|c|c|}
\hline Polymer/Solvent Fraction & $\begin{array}{l}\text { Polymer } \\
\text { Concentration }\end{array}$ & Viscosity & Porosity \\
\hline & g.cm ${ }^{-3}$ & cP & $\%$ \\
\hline $\begin{array}{l}\text { 1g P(VDF-TrFE) / } 4 \mathrm{~mL} \\
\text { DMF }\end{array}$ & 0,25 & 956,6 & 70 \\
\hline $\begin{array}{l}\text { 1g P(VDF-TrFE }) / 5 \mathrm{~mL} \\
\text { DMF }\end{array}$ & 0,20 & 629,3 & 72 \\
\hline $\begin{array}{l}\text { 1g P(VDF-TrFE }) / 6.7 \mathrm{~mL} \\
\text { DMF }\end{array}$ & 0,15 & 267,6 & 76 \\
\hline $\begin{array}{l}\text { 1g P(VDF-TrFE }) / 10 \mathrm{~mL} \\
\text { DMF }\end{array}$ & 0,10 & 81,2 & 80 \\
\hline
\end{tabular}

3. Results

After polymer dissolution, isothermal evaporation at different temperatures from 20 to $60^{\circ} \mathrm{C}$ was performed in an oven in order to obtain $\mathrm{P}(\mathrm{VDF}-\mathrm{TrFE})$ membranes and to study the influence of the polymer concentration and evaporation temperature and rate on the sample morphology.

\section{a) Thermogravimetric analysis}

In the TGA experiments the weight-loss, $\alpha$, is given by $[15,16]$ : 
$\alpha=\frac{w_{0}-w_{(t)}}{w_{0}-w_{\infty}}$

Eq. 2

where $w_{0}, w t_{(t)}$ and $w_{\infty}$ are the weights of the sample before solvent evaporation, at a given time $t$ and after complete drying, respectively. An analysis of the kinetics of solvent evaporation can be performed in terms of the general equation [16]:

$$
\frac{d \alpha}{d t}=k(T) f(x)=
$$

where $f(\alpha)$ is the reaction model, $k(T)$ is the temperature dependent rate constant, $T$ is the temperature and $t$ is the time. The rate constant is assumed to obey the Arrhenius equation:

$$
k(T)=Z \exp \left(-\frac{E}{R T}\right)
$$

where $Z$ is the pre-exponential factor, $E$ is the activation energy and $R$ is the ideal gas constant. If the process is a simple $n^{\text {th }}$ order reaction, the conversion dependence can be written as:

$$
f(\alpha)=(1-\alpha)^{n}
$$


Substituting equation 4 and 5 into equation 3 and taking logarithm, equation 6 is obtained:

$$
\ln \left(\frac{d \alpha}{d t}\right)=\ln (Z)+n \ln \left(-\alpha-\frac{E}{R T}\right.
$$

In the case of isothermal evaporation, this equation allows to determine the kinetics order $n$ of the solvent evaporation and the activation energy. The weight loss of the $\mathrm{P}(\mathrm{VDF}-\mathrm{TrFE})$ sample during the isothermal experiments was measured for temperatures between 20 and $60{ }^{\circ} \mathrm{C}$.

Figure 1.a shows the isothermal TGA traces at different temperatures obtained for the 5/95 P(VDF-TrFE)/DMF solution at different temperatures and figure $1 \mathrm{~b}$ the solvent weight loss conversion factor for the same sample. Figure $1 \mathrm{c}$ shown the solvent weight loss, $\alpha$ at $20^{\circ} \mathrm{C}$ for the $\mathrm{P}(\mathrm{VDF}-\mathrm{TrFE})$-DMF solutions at different initial concentrations.

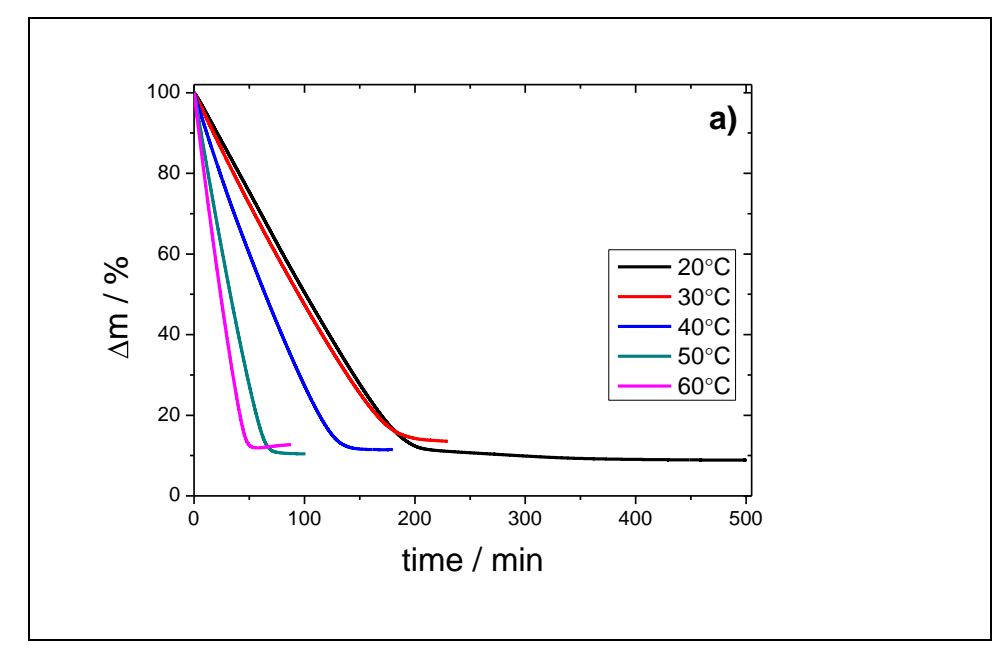




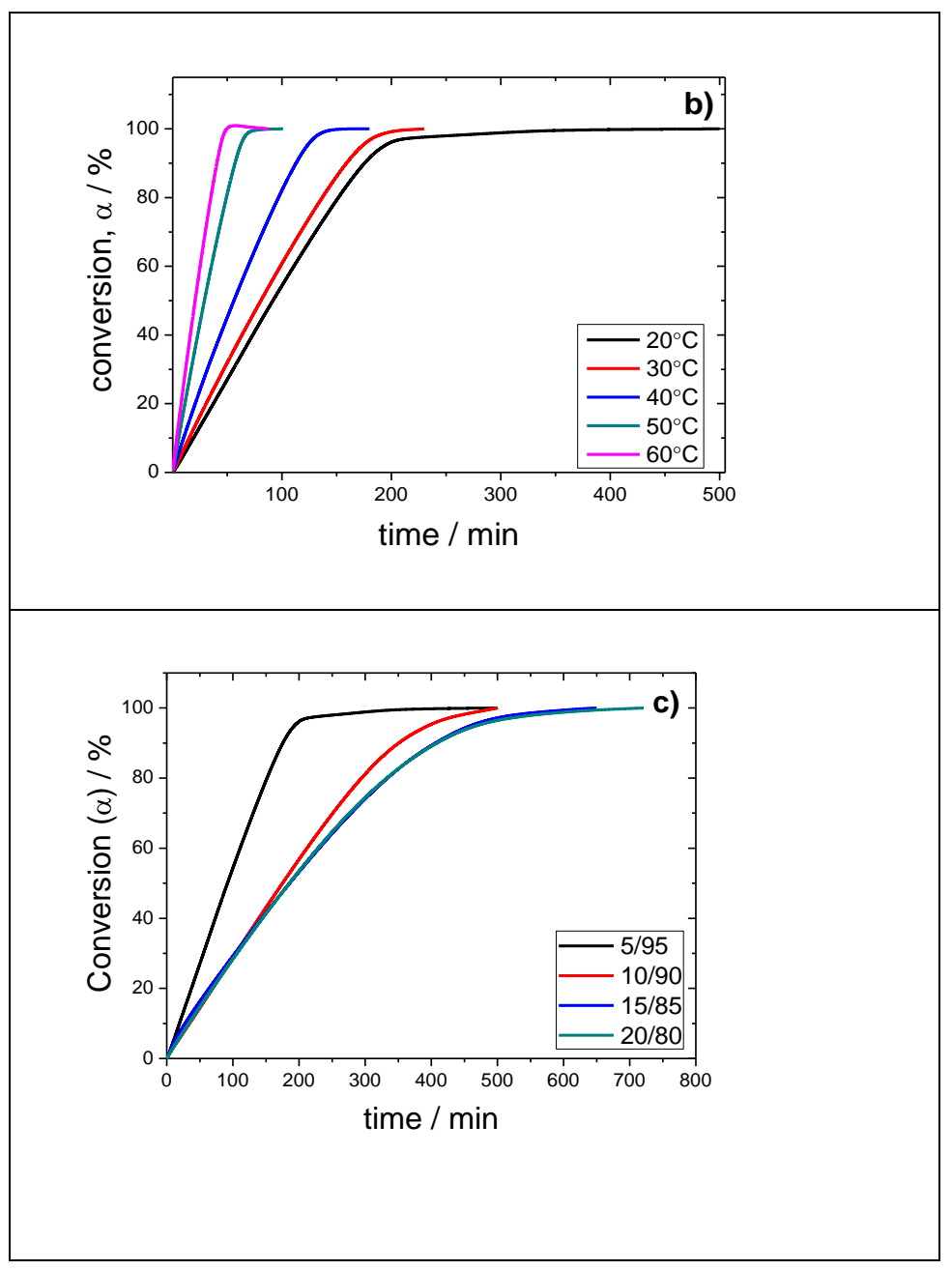

Figure 1 - a) Weight loss curves and b) solvent weight loss, $\alpha$ for the P(VDF-

TrFE)/DMF solution at several isothermal temperatures for the solution 5/95 c) Solvent weight loss, $\alpha$ at $20^{\circ} \mathrm{C}$ for the $\mathrm{P}(\mathrm{VDF}-\mathrm{TrFE}) / \mathrm{DMF}$ solutions of varying initial concentration

Figure 1 shows that after complete solvent evaporation a weight plateau was reached, corresponding to the mass of the polymer initially present in the solution. This plateau is reached more quickly as the isothermal temperature increases, as the evaporation of the solvent is faster for higher temperatures. The evolution of the $\mathrm{P}(\mathrm{VDF}-\mathrm{TrFE}) / \mathrm{DMF}$ weight loss conversion factor $(\alpha)$ is represented in figure $1 \mathrm{~b}$ for the different 
temperatures and in figure 1c for the different initial concentrations at a given temperature. The graphs presented in figure 1 are representative of the obtained results for the rest of the polymer/solvent ratios.

Drying of the polymer solution in the nitrogen flow atmosphere of the thermogravimeter can be also seen as a desorption process, governed by the diffusion coefficient of the solvent in the solution. In fact accepting a Fickian desorption kinetics, at least at the beginning of evaporation, the diffusion coefficient could be estimated from the slope of the $\alpha$ against the squared root of time. The plots of Figure 1 show that diffusion coefficient of the solvent, as corresponds to a thermally activated process, decreases with increasing temperature, but also that an increase of initial concentration of the solution hinders solvent diffusion and so decreases the initial evaporation rate. The time derivative of the weight loss curves (figure 2) reveals that the maximum rate of evaporation occurs at the initial steps of the measurements, right before each isothermal temperature is reached. Furthermore, the derivative of the weight loss curves is larger for higher temperatures, showing the increase of the evaporation rate with increasing temperature, while it decreases with time due to the progressive increase of solvent concentration during the evaporation process. This behaviour was already observed in the homopolymer PVDF [15].

Figure 2.a shows that the weight-loss rate $(d \alpha / d t)$ as a function of time shows two different regimes. The transition to the second regime in which the solvent evaporation rate rapidly collapses can be associated to liquid-liquid phase separation, consisting in the segregation of solvent from a polymer-rich liquid phase and further crystallization of the polymer to give the morphology shown by the SEM pictures of Figure 6 (see later the discussion about morphology evolution). The solvent evaporation rate in the second 
regime decreases faster as temperature increases (Figure 2a) or initial polymer concentration in the solution decreases (Figure 2b).

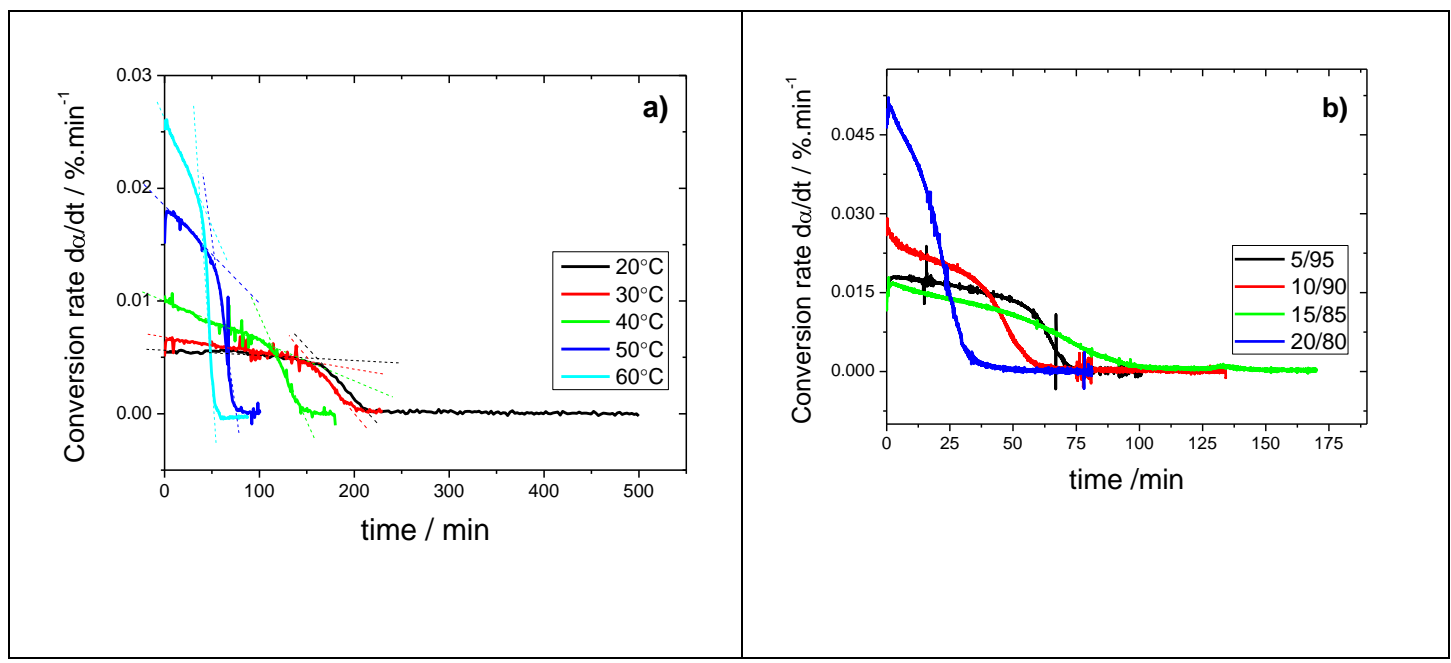

Figure 2 - Time dependence of the weight-loss rate $(d \alpha / d t)$ for the P(VDF-

TrFE)/DMF: a) $5 / 95$ at different isothermal temperatures, b) evaporated at $50{ }^{\circ} \mathrm{C}$ for different initial polymer/solvent concentrations.

In order to properly quantify the process, the plots of $\ln (d \alpha / d t)$ against $\ln (1-\alpha)$ for isothermal evaporation (Figure 3) can be fitted by linear relations and correlated using the $\mathrm{n}^{\text {th }}$ order method (Pearson $R^{2}>93 \%$ confidence). In this way, the reaction order $\mathrm{n}$ can be obtained ("general rate expression", equation 6). The values obtained for the reaction order of the $\mathrm{P}(\mathrm{VDF}-\mathrm{TrFE}) / \mathrm{DMF}$ system evaporation under isothermal conditions varies from 0.14 to 0.18 with increasing temperatures from 20 to $60{ }^{\circ} \mathrm{C}$. Chen et al. $[17,18]$ proposed a model for drying processes called the Reaction Engineering: in this approach, evaporation appears as a zero order thermally activated process, a feature that is coincident with our finding for the solvent evaporation. 


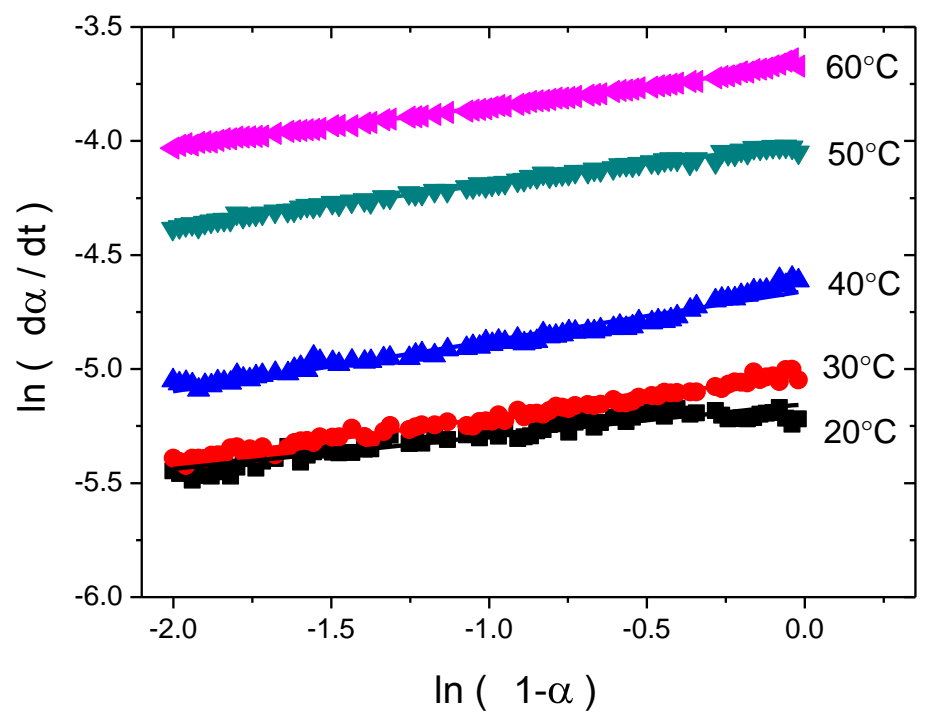

Figure $3-\ln (d \alpha / d t)$ against $\ln (1-\alpha)$ plots for isothermal evaporation for the P(VDFTrFE)/DMF 95/5 solution measured at different temperatures.

The determination of the kinetic parameters over a series of weight losses has more accuracy than the method of the "general rate expression". Flynn developed a method to calculate the kinetic parameters at different conversion levels using isothermal techniques [10-19]:

$\ln (t)=\ln (f(\alpha))-\ln (A)+\frac{E_{a c t}}{R T}$ Eq. 7

In equation 7, $t$ is time, $f(\alpha)$ is constant (calculations will be performed at different $\alpha$ intervals), $A$ is a constant, $R$ is the ideal gas constant, $T$ is the temperature of isothermal measurement and $E_{a c t}$ is the activation energy. Equation 7 also provides a way of calculating $E_{a c t}$ if the evaporation mechanism remains unchanged during the isothermal 
experiment [20]. This method is based on the slope of a plot of natural logarithm of time $v s$ inverse absolute test temperature, as presented in figure $4 \mathrm{a}$. .

Data presented in figure 4.a shows good linearity. The linear correlation coefficients obtained by fitting to equation 7 are higher than 0.90 and the slope of these linear fittings (Figure 4.a) remains constant with the increase of weight loss $\alpha$ over the range $30-90 \%$. This indicates that activation energy is independent of the conversion ratio, as represented in figure 4.b. The obtained results show a strong dependence of the $E_{\text {act }}$ with the initial polymer/solvent concentration. The values found for the activation energies are constant for the various conversion rates, for the same $\mathrm{P}(\mathrm{VDF}-\mathrm{TrFE}) / \mathrm{DMF}$ concentration, while in the case of the homopolymer PVDF the activation energy increases with the increase of $\alpha$ [15]. Furthermore, when compared the $E_{a c t}$ for the different polymer/solvent concentrations, the activation energy increases with the decrease of the amount of solvent present in the system. Moreover, for the same $\alpha$ it was observed a linear dependence of the $E_{a c t}$ with the polymer concentration (figure 5).
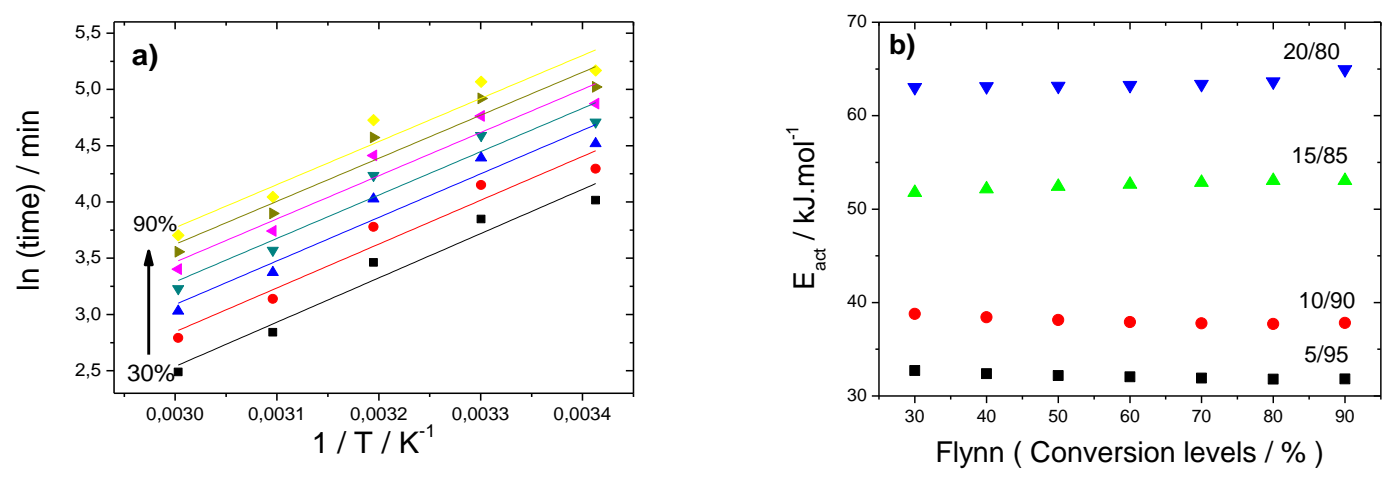

Figure 4 - a) Estimation of activation energy by the Flynn method for the P(VDFTrFE)/DMF (5/95) under isothermal conditions and b) evolution of the activation energy obtained by the Flynn method for different levels of conversion for the polymer/solvent concentrations of the samples obtained at room temperature. 


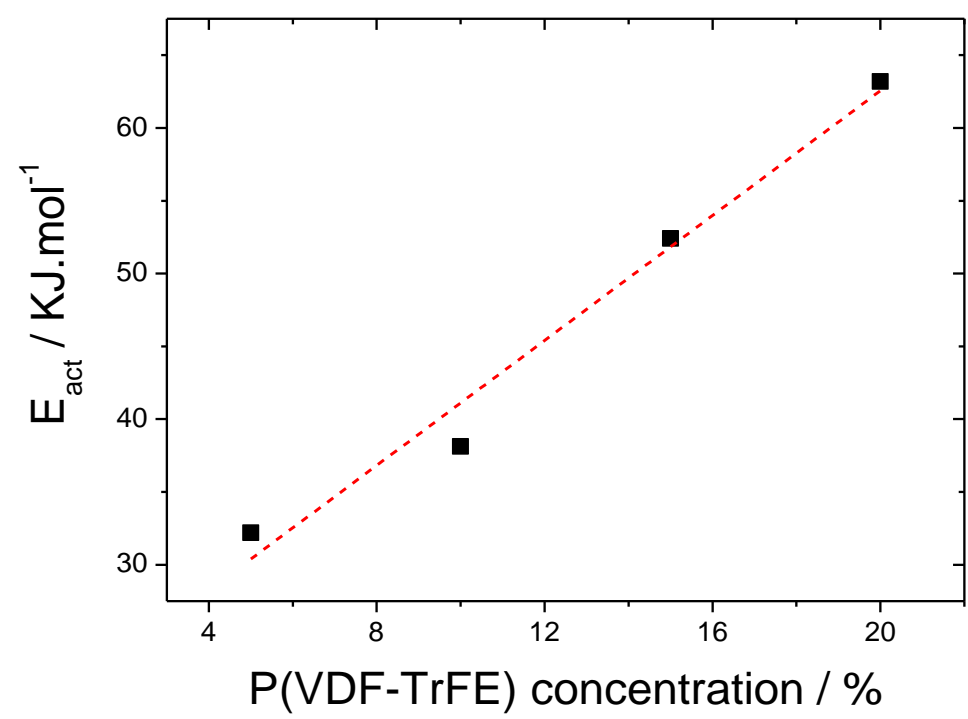

Figure 5 - Evolution of the activation energy with $\mathrm{P}(\mathrm{VDF}-\mathrm{TrFE})$ concentration present in the polymer/solvent system. The figure corresponds to $\alpha=50 \%$.

After complete crystallization of the polymer, the samples morphology was characterized by SEM in order to study the evolution of the pore structure. The obtained results are presented in figures 6 and 7.

In general, the crystallized material at room temperature and for decreasing polymer concentration presents the highest porosity and the porosity decreases with increasing crystallization temperature. For all the samples crystallized at $20^{\circ} \mathrm{C}$, the pores are spherical and with diameter around 30-35 $\mu \mathrm{m}$ for the lower polymer concentration (5/95) and approximately $10-15 \mu \mathrm{m}$ for the sample with higher polymer content in the solution (80/20). The pore walls are formed by adhered microspheres with diameter of around $4 \mu \mathrm{m}$ (figure $6 \mathrm{~d}$, inset). The interconnectivity of the pores results from the spaces between the polymer microspheres that form the pore walls, while larger pore throats appear due to some defects in the structure. The membrane thickness does not 
have influence on the final microstructure of the membrane and the solvent diffusion is fast enough to make the solution concentration homogeneous across the sample during the evaporation process [13].

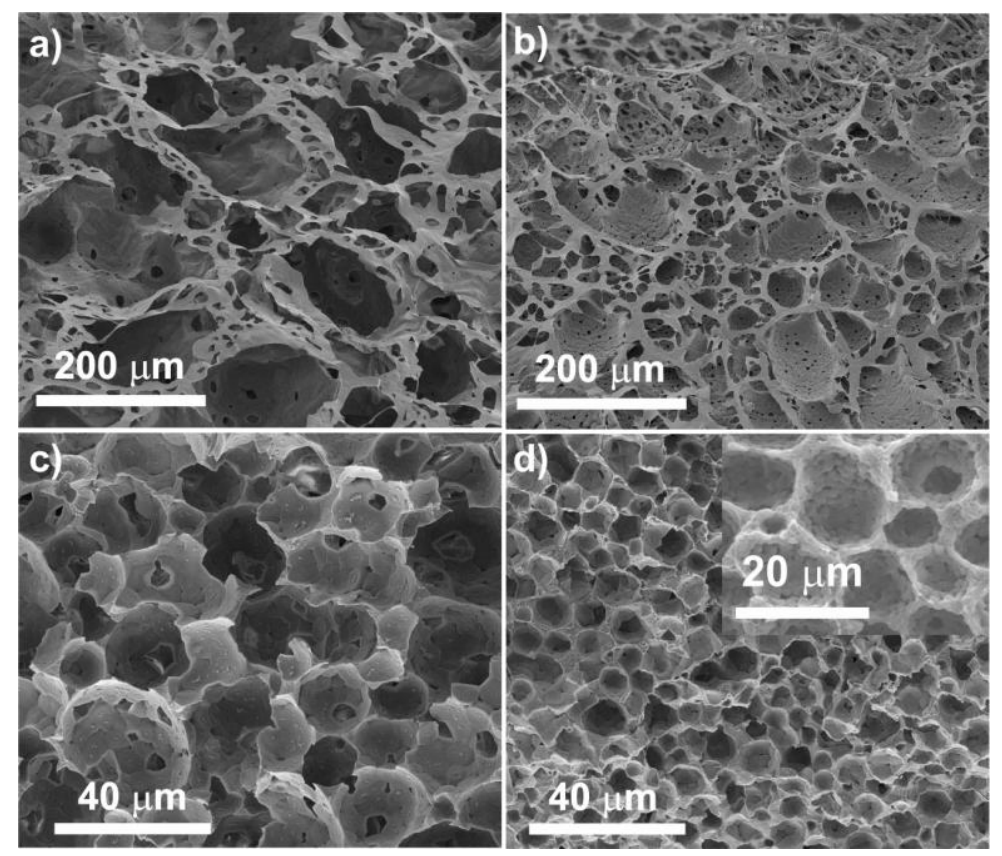

Figure 6 - SEM microphotographs of the surface and cross section $(a, b)$ for 5/95 $\mathrm{P}(\mathrm{VDF}-\mathrm{TrFE}) / \mathrm{DMF}$ and (c,d) 20/80 P(VDF-TrFE)/DMF samples obtained by solvent evaporation at room temperature.

For samples crystallized at $60^{\circ} \mathrm{C}$, a spherulitic structure similar to the one obtained for the PVDF homopolymer $[8,21]$ was observed (Figure 7 ), independently of the polymer/solvent ratio. It was also observed some porosity between the spherulites that can be correlated to the sample crystallization kinetics. As the spherulite grows, more polymer in the liquid state will be joined to the crystallization interface and, at the end, when no liquid are present in the interface between spherulites, a open space will be left giving origin to the small pores appearing among the spherulites. 

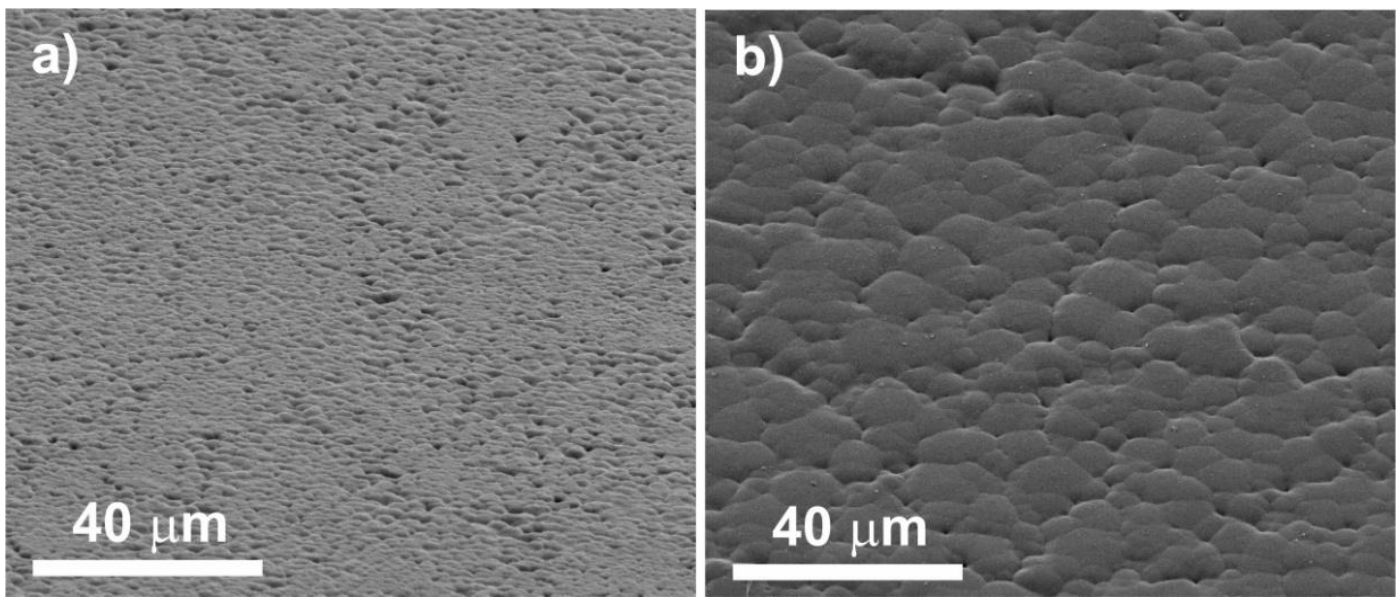

Figure 7 - SEM microphotographs of the samples surface crystallized at $60^{\circ} \mathrm{C}$ : a) $\mathrm{P}(\mathrm{VDF}-\mathrm{TrFE}) / \mathrm{DMF}(10 / 90)$ and b) P(VDF-TrFE)/DMF (20/80).

In figure 8 the liquid/liquid phase diagram for (PVDF-TrFE)/DMF solution at different crystallization temperatures (between 20 and $60^{\circ} \mathrm{C}$ ) and different polymer/solvent ratio is presented. 


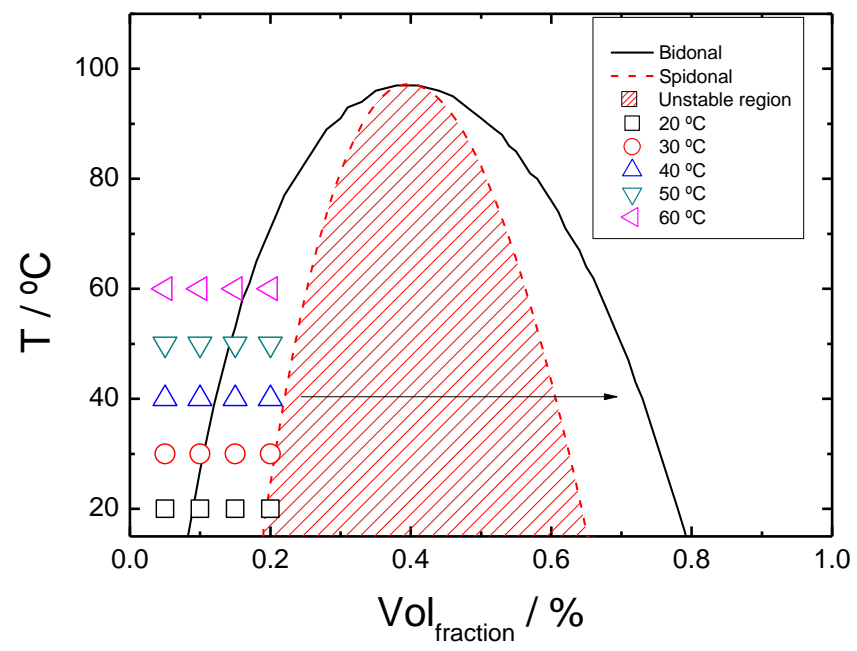

Figure 8 - Phase diagram for the $\mathrm{P}(\mathrm{VDF}-\mathrm{TrFE}) / \mathrm{DMF}$ solution, the dashed line corresponds to the initial volume faction for the different temperatures. The arrow represents the system evolution due to solvent evaporation.

In this figure the initial experimental conditions (temperature and volume fraction) are indicated (open dots) and also the shift toward higher volume fractions due to the isothermal solvent evaporation (arrow). This evolution can produce polymer crystallization if the state of the system falls into a liquid/solid binary region, before a liquid/liquid phase separation takes place.

The morphology shown in figures 6 and 7 indicates a spidonal liquid/liquid phase separation and are according to what was found in PVDF homopolymer membranes produced by TIPS from a solution in a solvent/nonsolvent mixture [7, 10, 12, 13, 22]. Crystallization process of the $\mathrm{P}(\mathrm{VDF}-\mathrm{TrFE})$ is quite different from that obtained for the PVDF due to the irregularity introduced in the polymer chain by the TrFE groups [23]. The observed shift to the crystallization line in the phase diagram with respect to the 
PVDF makes the liquid /liquid binary region to appear before crystallization takes place. Further, the solvent evaporation produces $\mathrm{P}(\mathrm{VDF}-\mathrm{TrFE})$ crystallization.

The shape of the phase diagram in the region of the liquid/liquid coexistence region can be predicted with the help of the Flory-Huggins (FH) lattice teory [13].

In the phase diagram (figure 8) two mechanisms have to be considered: nucleation and growth (NG) and spinodal decomposition (SD). The first one occurs in a metastable region of the phase diagram between spinodal and binodal lines, while SD occurs in a unstable region under spinodal lines. In the SD region, small variations in the composition destabilizes the system, separating it into two phases with lower free energy. In the metastable region a large flutuation in the composition is needed to create a domain larger than some crtical ratio, in order to enable the NG process [13, 24]. In the PVDF-TrFE copolymer, the pattern is formed by an initial low concentration that will put the system in the metastable region of the phase diagram (figure 9). As the solvent evaporates, the polymer concentration will enter in the unstable region of the phase diagram. Here the grain boundary (GB) constructs the observed bands, supressing the grains growth while in the interior the SD continues. As the system continues to evolve in the phase diagram to the metastable region (nucleation and growth) the GB effects decrease and the interior of the grain have the circular shape predicted by the Cahn-Hilliard equation for mass conservation systems [13, 25, 26], which implies that inside the grain the mass is conserved.

Finally, two factors have to be considered to the formation of such patterns: a) initial volume fration of the polymer/solvent and b) the initial position in the metastable region. Volume fraction near the binodal line are highly affected by the NG process leading to close porous; volume fraction near the spinodal will not be affect by the initial NG, leading to structures without the grain growth. 
The differences in the final microstructure found among the samples crystallized at different temperatures are related to the polymer concentration in the solution when liquid-liquid transition takes place and the solvent evaporation rate changes. The former can be calculated by extrapolation of the two regimes straight lines in the evaporation rate against time plot of Figure 3. The intersection allows estimating the time onset of phase separation and this value allows calculating, with the data of Figure 2, the polymer concentration at that moment. It is found that by increasing initial polymer concentration in the solution, the polymer/solvent ratio at the phase separation onset increases. Thus at $20^{\circ} \mathrm{C} \mathrm{P}(\mathrm{VDF}-\mathrm{TrFE}) / \mathrm{DMF}$ ratio is $22 / 78,28 / 72,39 / 61,47 / 53$ at the phase separation when the initial concentrations are 5/95, 10/90, 15/85 and 20/80 respectively. Slower solvent evaporation and more diluted solution when phase separation takes place yields a more mobile polymer-rich phase and, as shown in Figure 6, smaller aggregates of the liquid solvent segregated. On the other hand, as temperature increases, this phenomenon is more pronounced, and in fact at $60^{\circ} \mathrm{C}$ nearly no presence of the macropores originated in liquid-liquid phase separation are observed in the dry material. The structure is then nearly the same than that produced in PVDF by liquidsolid phase transition [15].

The nature of the crystalline phase present in the polymer can be identified by DSC and FTIR. FTIR spectra for the samples crystallized at room temperature shows that the crystallization of the polymer occurs in the ferroelectric phase (figure 9a) due to the presence of the characteristic absorption band at $840 \mathrm{~cm}^{-1}[2,27]$. 

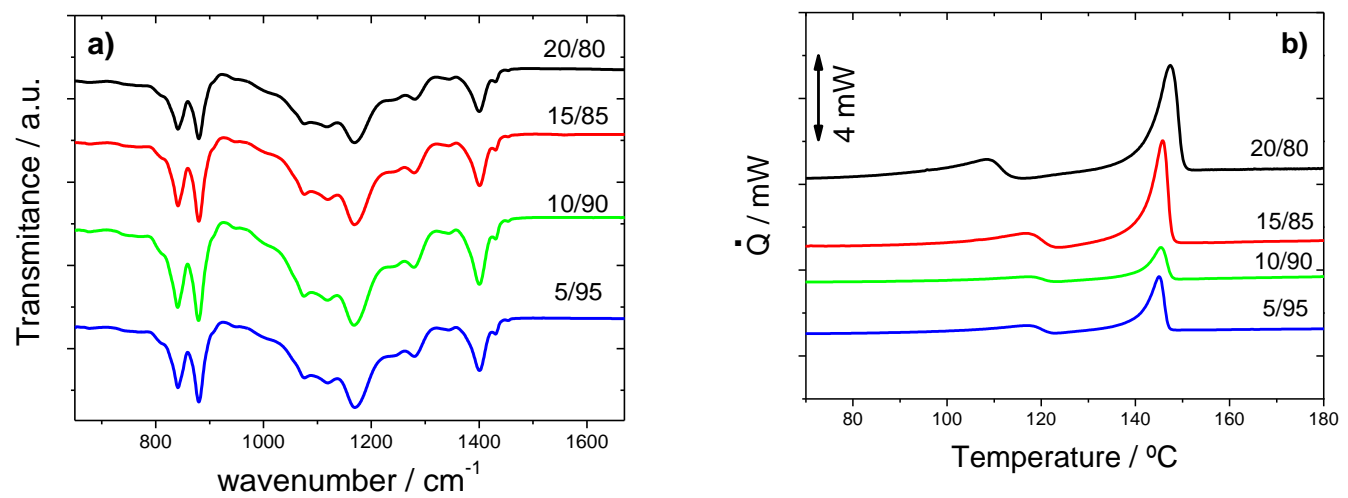

Figure 9-a) Infrared measurements for the PVDF-TrFE samples obtained by room temperature crystallization from different $\mathrm{P}(\mathrm{VDF}-\mathrm{TrFE}) / \mathrm{DMF}$ solutions and $\mathbf{b}) \mathrm{DSC}$ curves for the same samples.

In the DSC thermograms (figure 9b) two peaks are observed, the one that occurs at the lower temperature corresponds to the ferroelectric-paraelectric transition (FE-PE) with maxima at ca. $117^{\circ} \mathrm{C}$. The higher DSC endotherm corresponds to the melting of the paraelectric phase and it located at ca. $145^{\circ} \mathrm{C}$. It can be observed that the temperature or initial concentration in the crystallization process does not affect the FE-PE transition temperature or the melting temperature of the polymer, what leads to the conclusion that solvent evaporation rate does not play an important role in the final polymer crystalline properties, but in the crystallization dynamics, which affects the porosity and consequently the microstructure of the $\mathrm{P}(\mathrm{VDF}-\mathrm{TrFE})$ membranes depending on the specific place of the polymer/solvent solution phase diagram in which the isothermal crystallization begins. 


\section{Conclusions}

Ferroelectric macroporous poly(vinylidene fluoride-trifuoroethylene) membranes with tailored interconnected porosity and with great potential for biomedical and energy applications have been produced by isothermal crystallization from the solution at different temperatures starting from different diluted solutions of the co-polymer in dimethylformamide.

It is concluded that the temperature or initial concentration of the crystallization process does not affect neither the phase content of the polymer, nor its ferroelectric transition temperature or the melting temperature of the polymer. In this way, the solvent evaporation rate does not play an important role in the final polymer crystalline properties, but in the crystallization dynamics, which affects the porosity and the microstructure of the $\mathrm{P}(\mathrm{VDF}-\mathrm{TrFE})$ membranes.

The microstructure depends on the specific place of the polymer/solvent solution phase diagram in which the isothermal crystallization begins. The shape of the phase diagram in the region of the liquid/liquid coexistence region can be predicted with the help of the Flory-Huggins $(\mathrm{FH})$ lattice teory and for the patterning formation two mechanisms have to be considered: nucleation and growth and spinodal decomposition. These mechanism, toghether with the Cahn-Hilliard equation for mass conservation systems give a theoretical explanation of the observed microstructural evolution along the phase diagram.

\section{Acknowledgements}

The authors thank the Portuguese Foundation for Science and Technology (FCT) Grants PTDC/CTM-NAN/112574/2009, PTDC/CTM/73030/2006, PTDC/CTM/69316/2006 
and NANO/NMed-SD/0156/2007. V.F., C.M.C. and V.S. thank the FCT for the

SFRH/BD/44289/2008, SFRH/BD/68499/2010, SFRH/BPD/63148/2009 grants,

respectively. JLGR acknowledge the founding form the Programa de Apoyo a la

Investigación y Desarrollo (PAID-00-09) of the Universidad Politécnica de Valencia for a short stay in Universidade do Minho, Braga, the support of the Spanish Ministry of Science through project No. MAT2010-21611-C03-01 (including the FEDER financial support) and funding for research in the field of Regenerative Medicine through the collaboration agreement from the Conselleria de Sanidad (Generalitat Valenciana), and the Instituto de Salud Carlos III (Ministry of Science and Innovation). The authors wish also thank the CeNTI - Centre for Nanotechnology and Smart Materials, Rua Fernando Mesquita, 2782, 4760-034 Vila Nova de Famalicão, Portugal for allowing the use of some experimental equipment.

\section{References}

[1] Lovinger AJ. Developments in Crystalline Polymers. In: Basset DC, editor. London: Elsevier; 1982.

[2] Nalwa HS. Ferroelectric polymers: chemistry, physics and applications. New York: Marcel Dekker; 1995.

[3] Xiao Q, Wang X, Li W, Li Z, Zhang T, Zhang H. Macroporous polymer electrolytes based on PVDF/PEO-b-PMMA block copolymer blends for rechargeable lithium ion battery. Journal of Membrane Science. 2009;334(1-2):117-122.

[4] Ji G-L, Zhu B-K, Cui Z-Y, Zhang C-F, Xu Y-Y. PVDF porous matrix with controlled microstructure prepared by TIPS process as polymer electrolyte for lithium ion battery. Polymer. 2007;48(21):6415-6425.

[5] Laroche G, Marois Y, Schwarz E, Guidoin R, King MW, Pâris E, et al. Polyvinylidene Fluoride Monofilament Sutures: Can They Be Used Safely for Long-Term Anastomoses in the Thoracic Aorta? Artificial Organs. 1995;19(11):1190-1199.

[6] Klinge U, Klosterhalfen B, Öttinger AP, Junge K, Schumpelick V. PVDF as a new polymer for the construction of surgical meshes. Biomaterials. 2002;23(16):3487-3493.

[7] Han X, Ding H, Wang L, Xiao C. Effects of nucleating agents on the porous structure of polyphenylene sulfide via thermally induced phase separation. Journal of Applied Polymer Science. 2008;107(4):2475-2479.

[8] Sencadas V, Gregorio R, Lanceros-Méndez S. $\alpha$ to $\beta$ Phase Transformation and Microestructural Changes of PVDF Films Induced by Uniaxial Stretch. Journal of Macromolecular Science, Part B. 2009;48(3):514-525. 
[9] Ding Y, et al. Preparation of PVdF-based electrospun membranes and their application as separators. Science and Technology of Advanced Materials. 2008;9(1):015005.

[10] Su Y, Chen C, Li Y, Li J. PVDF Membrane Formation via Thermally Induced Phase Separation. Journal of Macromolecular Science, Part A: Pure and Applied Chemistry. 2007;44(1):99-104.

[11] Matsuyama H, Teramoto M, Kudari S, Kitamura Y. Effect of diluents on membrane formation via thermally induced phase separation. Journal of Applied Polymer Science. 2001;82(1):169-177.

[12] Yeow ML, Liu YT, Li K. Morphological study of poly(vinylidene fluoride) asymmetric membranes: Effects of the solvent, additive, and dope temperature. Journal of Applied Polymer Science. 2004;92(3):1782-1789.

[13] Ferreira A, Silva J, Sencadas V, Ribelles JLG, Lanceros-Méndez S. Poly[(vinylidene fluoride)co-trifluoroethylene] Membranes Obtained by Isothermal Crystallization from Solution. Macromolecular Materials and Engineering. 2010;295(6):523-528.

[14] Li L, Ding S, Zhou C. Preparation and degradation of PLA/chitosan composite materials. Journal of Applied Polymer Science. 2004;91(1):274-277.

[15] Magalhães R, Durães N, Silva M, Silva J, Sencadas V, Botelho G, et al. The Role of Solvent Evaporation in the Microstructure of Electroactive $\beta$-Poly(Vinylidene Fluoride) Membranes Obtained by Isothermal Crystallization. Soft Materials. 2010;9(1):1-14.

[16] Turi E. Thermal Characterization of Polymeric Materials. New York1997.

[17] Chen; XD, Xie GZ. Fingerprints of the drying behaviour of particulate or thin layer food materials established using a reaction engineering approach. Trans IChemE Part C: Food Bioprod Processing. 1997;75C:213-222.

[18] Chen XD. The Basics of a Reaction Engineering Approach to Modeling Air-Drying of Small Droplets or Thin-Layer Materials. Drying Technology. 2008;26(6):627-639.

[19] Flynn JH. Thermal Analysis. New York: Academic Press; 1969.

[20] Hamciuc C, Vlad-Bubulac T, Petreus O, Lisa G. Kinetics of thermal degradation in nonisothermal conditions of some phosphorus-containing polyesters and polyesterimides.

European Polymer Journal. 2007;43(3):980-988.

[21] Sencadas V, Gregorio Filho R, Lanceros-Mendez S. Processing and characterization of a novel nonporous poly(vinilidene fluoride) films in the $\beta$ phase. Journal of Non-Crystalline Solids. 2006;352(21-22):2226-2229.

[22] Cheng L-P, Lin D-J, Shih C-H, Dwan A-H, Gryte CC. PVDF membrane formation by diffusioninduced phase separation-morphology prediction based on phase behavior and mass transfer modeling. Journal of Polymer Science Part B: Polymer Physics. 1999;37(16):2079-2092.

[23] Gregorio R, Chaud MR, Nunes Dos Santos W, Baldo JB. Miscibility and morphology of poly(vinylidene fluoride)/poly[(vinylidene fluoride)-ran-trifluorethylene] blends. Journal of Applied Polymer Science. 2002;85(7):1362-1369.

[24] Bower DI. An Introduction to Polymer Physics. Cambridge: Cambridge University Press; 2002.

[25] Cahn JW. Phase Separation by Spinodal Decomposition in Isotropic Systems: AIP; 1965.

[26] Cahn JW, Hilliard JE. Free Energy of a Nonuniform System. I. Interfacial Free Energy: AIP; 1958.

[27] Simoes RD, Rodriguez-Perez MA, De Saja JA, Constantino CJL. Tailoring the structural properties of PVDF and P(VDF-TrFE) by using natural polymers as additives. Polymer Engineering \& Science. 2009;49(11):2150-2157. 


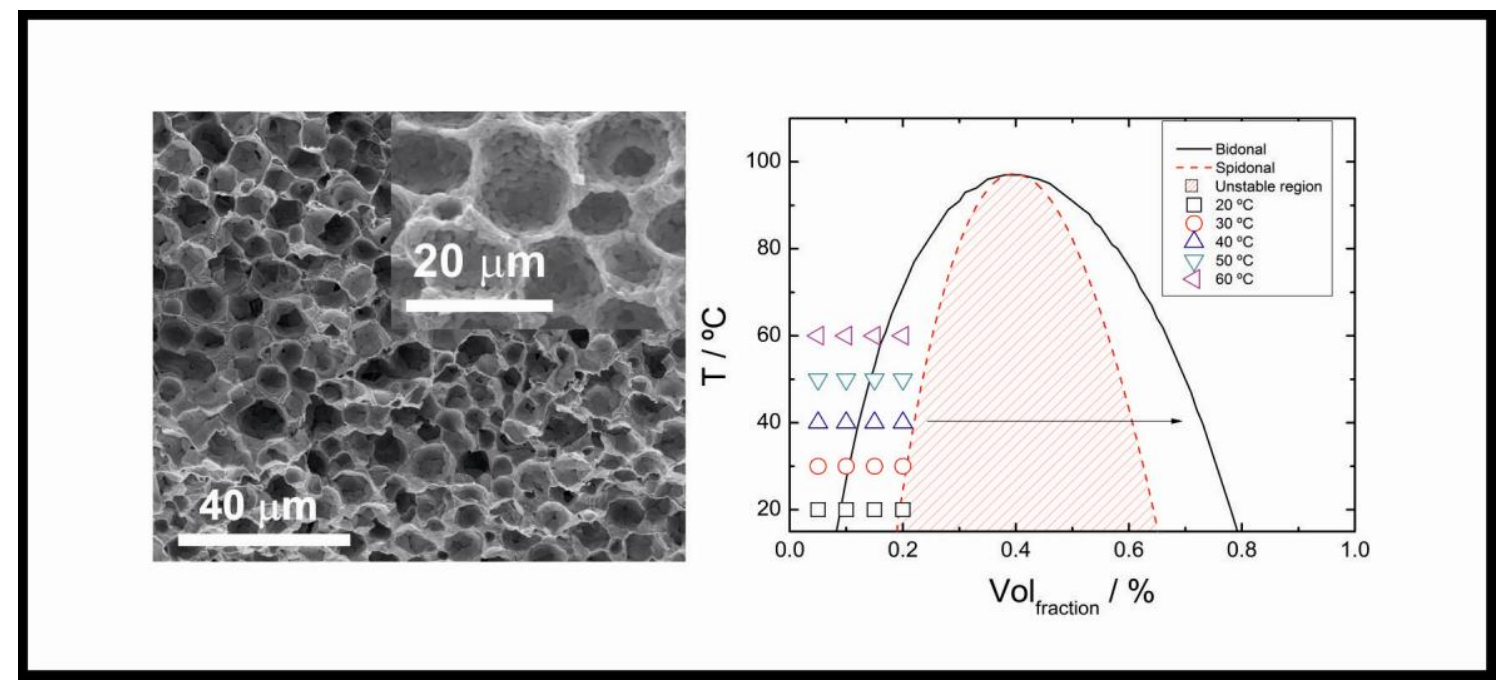

